OPEN ACCESS

Edited by:

Laurent Gautron,

University of Texas Southwestern Medical Center, United States

Reviewed by:

Patricia Jusuf,

University of Melbourne, Australia

Stephan C. F. Neuhauss,

Universität Zürich, Switzerland

*Correspondence:

Julio D. Amigo

jamigo@bio.puc.cl

Received: 06 December 2017 Accepted: 12 March 2018 Published: 27 March 2018

Citation:

Stanic K, Quiroz A, Lemus CG Wichmann IA, Corvalán AH, Owen GI, Opazo JC, Concha ML and Amigo JD (2018) Expression of RPRM/rprm in the Olfactory System of Embryonic Zebrafish (Danio rerio).

Front. Neuroanat. 12:23.

doi: 10.3389/fnana.2018.00023

\section{Expression of RPRM/rprm in the Olfactory System of Embryonic Zebrafish (Danio rerio)}

\author{
Karen Stanic ${ }^{1}$, Alonso Quiroz ${ }^{1}$, Carmen G. Lemus ${ }^{2}$, Ignacio A. Wichmann ${ }^{3,4}$, \\ Alejandro H. Corvalán ${ }^{3,4,5}$, Gareth I. Owen ${ }^{1,3,4,5,6}$, Juan C. Opazo ${ }^{7}$, Miguel L. Concha ${ }^{2,8}$ and \\ Julio D. Amigo ${ }^{1 *}$ \\ ${ }^{1}$ Departamento de Fisiología, Facultad de Ciencias Biológicas, Pontificia Universidad Católica de Chile, Santiago, Chile, \\ ${ }^{2}$ Anatomy and Developmental Biology Program, Faculty of Medicine, Institute of Biomedical Sciences, Universidad de Chile, \\ Santiago, Chile, ${ }^{3}$ Advanced Center for Chronic Diseases, Santiago, Chile, ${ }^{4}$ UC Center for Investigation in Oncology, \\ Pontificia Universidad Católica de Chile, Santiago, Chile, ${ }^{5}$ Departamento de Oncología y Hematología, Facultad de Medicina, \\ Pontificia Universidad Católica de Chile, Santiago, Chile, ${ }^{6}$ Millennium Institute on Immunology and Immunotherapy, Santiago, \\ Chile, ${ }^{7}$ Instituto de Ciencias Ambientales y Evolutivas, Facultad de Ciencias, Universidad Austral de Chile, Valdivia, Chile, \\ ${ }^{8}$ Biomedical Neuroscience Institute, Universidad de Chile, Santiago, Chile
}

The Reprimo (RPRM) family is composed of highly conserved single-exon genes. The expression pattern of this gene family has been recently described during zebrafish (Danio rerio) embryogenesis, and primarily locates in the nervous system. Its most characterized member, RPRM, which duplicated to give rise rprma and rprmb in the fish lineage, is known to act as a tumor-suppressor gene in mammalian models. Here, we describe in detail the spatiotemporal expression of three rprm genes (rprma, rprmb, and rprml) within distinct anatomical structures in the developing peripheral and central nervous system. In the zebrafish, rprma mRNA is expressed in the olfactory placodes (OP) and olfactory epithelium (OE), rprmb is observed in the tectum opticum (TeO) and trigeminal ganglion (Tg), whereas rprm/ is found primarily in the telencephalon (Tel). At protein level, RPRM is present in a subset of cells in the OP, and neurons in the $\mathrm{OE}, \mathrm{TeO}$, hindbrain and sensory peripheral structures. Most importantly, the expression of RPRM has been conserved between teleosts and mammals. Thus, we provide a reference dataset describing the expression patterns of RPRM gene products during zebrafish and mouse development as a first step to approach the physiological role of the RPRM gene family.

Keywords: reprimo, zebrafish (Danio rerio), olfactory systems, opticum tectum, neurogenesis

\section{INTRODUCTION}

Reprimo is a family of poorly characterized single-exon genes composed by three paralogs: Reprimo (RPRM), RPRM-Like (RPRML), and RPRM3 (Wichmann et al., 2016). RPRM and $R P R M L$ have been differentially retained in most vertebrates, including humans, whereas RPRM3 has been retained only in a fraction of vertebrates, including teleost fish (Wichmann et al., 2016). RPRM is a highly glycosylated cytoplasmic protein that induces cell cycle arrest at G2 in a p53-dependent manner, by inhibiting Cdc2-cyclin B1 complex activity through an as yet unidentified cytoplasmic mechanism (Ohki et al., 2000; Taylor and Stark, 2001). RPRM expression is altered in several types of cancers (Sato et al., 2006; Xu et al., 2012; Saavedra et al., 2015), but there is no evidence to date for RPRM physiological function. 
Recently, we showed that $R P R M / r p r m$ genes are expressed in the zebrafish brain and that the expression domains are conserved between fish and mammals (Figueroa et al., 2017). Since teleost fish underwent an extra round of whole-genome duplication, zebrafish have retained duplicated copies of $R P R M$ genes: rprma and rprmb (Wichmann et al., 2016). Importantly, a precise temporal description of RPRM protein expression during the development of neuronal structures is still missing. Such knowledge is fundamental to advance in our understanding of the possible role of this gene family in neuronal development.

To this end, the zebrafish (Danio rerio) is an established model to study early nervous system development, which combines the complexity of a vertebrate organism with the easy-to-use and high-throughput capabilities of in vitro models (MacRae and Peterson, 2015). Moreover, zebrafish is very well-suited for systematic analysis of gene expression patterns at transcript level by whole-mount in situ hybridization (WISH), and at protein level by immunohistochemistry/immunofluorescence (IHC/IF). Additionally, many of the components that regulate developmental processes are evolutionarily conserved among vertebrate species (Saraiva et al., 2015; Hildebrand et al., 2017).

Herein we examine the temporal and spatial expression patterns of RPRM gene-products (mRNA and protein) during neural development. In zebrafish, rprm (rprma, rprmb) and rprml transcripts are expressed in confined regions of the embryonic nervous system including the olfactory placode (OP) and epithelium (OE). Our data constitutes the first evidence that $R P R M$ transcripts and proteins are expressed at early stages of $\mathrm{OP}$ and $\mathrm{OE}$ development. Furthermore, we show that $R P R M$ expression in the olfactory system is conserved between zebrafish and mouse. Our study using the zebrafish model system sets the basis to examine the functional role of the RPRM gene family in neural development.

\section{MATERIALS AND METHODS}

\section{Multiple Sequence Alignment}

We annotated RPRM genes in human, mouse and zebrafish. Complete amino acid sequences for the three paralogs in the three species were aligned using the L-INS-i strategy from MAFFT v.7 (Katoh and Standley, 2013). Potential domains were predicted using the TMHMM method (http:// www.cbs.dtu.dk/services/TMHMM/) as implemented in Geneius Software. To assess the potential cross-reactivity between human RPRM antibody and zebrafish Rprma/Rprmb proteins, the immunogenic sequence from human RPRM used to generate rabbit polyclonal anti-RPRM antibody (SAB1102454, SigmaAldrich Chemie $\mathrm{GmbH}$ ) was aligned against zebrafish Rprma and Rprmb using the same strategy described above.

\section{Zebrafish Maintenance and Husbandry}

Wild-type (TAB5) zebrafish (Danio rerio) were maintained according to standard methods (Westerfield, 1995). Embryos were raised in system water at $28^{\circ} \mathrm{C}$ and staged according to hours post-fertilization (hpf) and morphological criteria (Kimmel et al., 1995). All zebrafish studies were conducted under the guidance and approval of the Institutional Animal Care and Use Committee and the Bioethical Committee at Pontificia Universidad Católica de Chile.

\section{cRNA Probe Synthesis and Whole-Mount in Situ Hybridization (WISH)}

Templates for probe synthesis were PCR amplified from embryonic zebrafish cDNA using primers containing T7 RNA polymerase promoter sequence. To minimize cross-reactivity, the $5^{\prime}$ untranslated (5'-UTR) regions of $R P R M$ genes were used for primer design. Primer sets were designed as follows: rprma, fw: 5' - TGAGGAGAACCTCCTGTGCT-3', rv: 5' -TAATACGACT CACTATAGGGGCCTGATCCTGATGGTTCGT-3'; $r p r m b$, fw: 5' - TCCACCCATTCATCCTGTCA-3', rv: 5'-TAATACGACT CACTATAGGGTCGGAGTTTCTTCGTTTGTG-3'; and rprml, fw: 5-GACCGGAGATCATCCAAAGA-3', rv: 5' -TAATACGAC TCACTATAGGGCTCGTTTCGTAAACGTGCAA-3'. All PCR products were of the expected size as inspected by agarose gel electrophoresis (data not shown). Purified PCR products were in vitro transcribed and labeled using digoxigenin (DIG) RNA labeling Kit (Roche) according to manufacturer's protocol. cRNA probes were purified using mini Quick Spin RNA Columns (Roche) and stored at $-80^{\circ} \mathrm{C}$ with deionized formamide. WISH was carried out as described previously (Amigo et al., 2009). Embryos older than $24 \mathrm{hpf}$ were treated with $0.003 \%$ 1-phenyl 2-thiourea (Sigma) to inhibit pigmentation.

\section{Immunohistochemistry (IHC) Staining}

Zebrafish embryos at 24, 48, and 72 hpf were fixed in $4 \%$ paraformaldehyde or Trichloroacetic acid (TCA -depending of developmental stage-) $2 \%$ overnight at $4^{\circ} \mathrm{C}$. Fixed embryos were washed in PBT (1\% Triton $\mathrm{x}-100)$ and subsequently treated with acetone for $20 \mathrm{~min}$ at $-20^{\circ} \mathrm{C}$, washed and consecutively treated with either Proteinase K (10 ng/mL; 24-48 hpf embryos) or trypsin $1 \mathrm{x}$ (72 hpf embryos). Blocking was carried out with $10 \%$ FBS $+1 \%$ DMSO in PBT twice for $1 \mathrm{~h}$ each and treated overnight with $0.1 \% \mathrm{H}_{2} \mathrm{O}_{2}$. Embryos were then washed in PBT and incubated with primary antibody at $4^{\circ} \mathrm{C}$ for 3 days with mild shaking. Antibody dilution was prepared in blocking solution containing $0.2 \%(\mathrm{w} / \mathrm{v}$ ) of blocking reagent (Roche). Rabbit polyclonal anti-RPRM antibody (SAB1102454, Sigma-Aldrich Chemie $\mathrm{GmbH}$ ) and mouse monoclonal anti-acetylated tubulin (T6793, Sigma-Aldrich Chemie $\mathrm{GmbH}$ ) were used in 1:800 and 1:1000 dilutions respectively; rabbit policlonal anti-GFP (A11122, Thermo Fisher Scientific) was used in 1:500 dilution. For secondary antibody labeling, goat anti-rabbit IgG Alexa-488 and goat anti-mouse IgG Alexa-546 (Invitrogen) were used at 1:200 dilution in blocking buffer for $2 \mathrm{~h}$ at room temperature in combination with Hoechst 33342 nuclear marker at $1 \mu \mathrm{g} / \mathrm{mL}$ (ThermoFisher Scientific Inc.).

\section{Knockdown of RPRM by Morpholino (MO) Microinjections}

Embryos were microinjected at 1-2 cell-stage with $3 \mathrm{~nL}$ of morpholino solution (along with phenol red) at a concentration of $1 \mathrm{mM}$ for RPRM (rprma: 5'-AGTCCAAGGCTTCAGACA GTGTCGT-3' and rprmb: 5'-AATTCATGCTGAACTGCTGTT 
CTCT- $\left.3^{\prime}\right)$ and $0.3 \mathrm{mM}$ for standard control morpholino (5'CCTCTTACCTCAGTTACAATTTATA-3'). RPRM-MOs were obtained from Gene Tools (Gene Tools, LLC) and designed to block the translation start site. Reduction in protein synthesis was confirmed by immunohistochemistry (IHC) when compared $R P R M-\mathrm{MO}$ injected embryos with controls.

\section{Imaging}

After WISH, zebrafish embryos were embedded in $75 \%$ glycerol/PBS and imaged using NIKON eclipse $80 \mathrm{i}$ microscope equipped with a DS-Vi1 (NIKON) camera. For fluorescent whole mount immunohistochemistry, embryos were mounted in acrylic rings with glass bottoms containing $1 \%$ low melting point agarose and images were acquired using Leica TCS LSI macro zoom confocal microscope.

\section{RESULTS}

\section{Expression Patterns of rprm (rprma/rprmb) and rprml During Zebrafish Neural Development}

Expression profiles of rprma, rprmb and rprml in the head region of embryos were analyzed by WISH from 24 until $72 \mathrm{~h}$ postfertilization (hpf) (Figures 1, 2). At $24 \mathrm{hpf}$, rprma is expressed

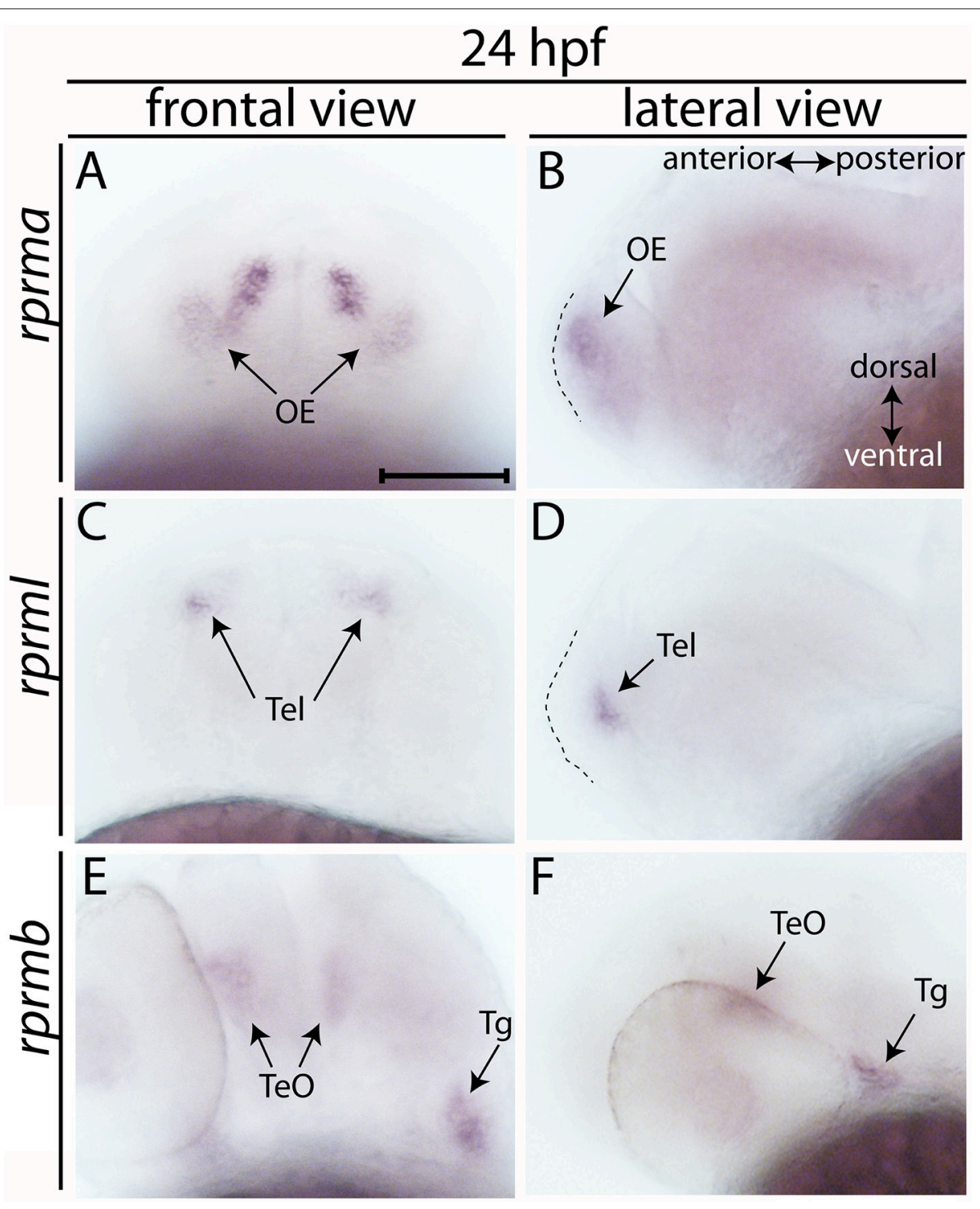

FIGURE 1 | Expression of RPRM mRNA at $24 \mathrm{~h}$ post-fertilization (hpf). (A-E) The expression patterns of rprma/b and rprm/ were visualized by whole-mount in situ hybridization (WISH) during zebrafish early neuronal development. (A,C,E) Frontal views of the embryos heads, with dorsal to the top and ventral to the bottom (double arrow in $\mathbf{B})$. (B,D,F) Lateral views of the embryos head with anterior to the left and posterior to the right (double arrow in $\mathbf{B})$. (B,D) dashed areas represent the most anterior part of the embryo's head. At 24 hpf (A,B) rprma, (C,D) rprml, and (E,F) rprmb transcripts are located in neuronal cell populations such as: (A,B) olfactory placode (OP, black arrows), (C,D) telencephalon (Tel, black arrows), and (E,F) tectum opticum (TeO) and trigeminal ganglia (Tg), respectively. Scale bar in (A): $100 \mu \mathrm{m}$. 

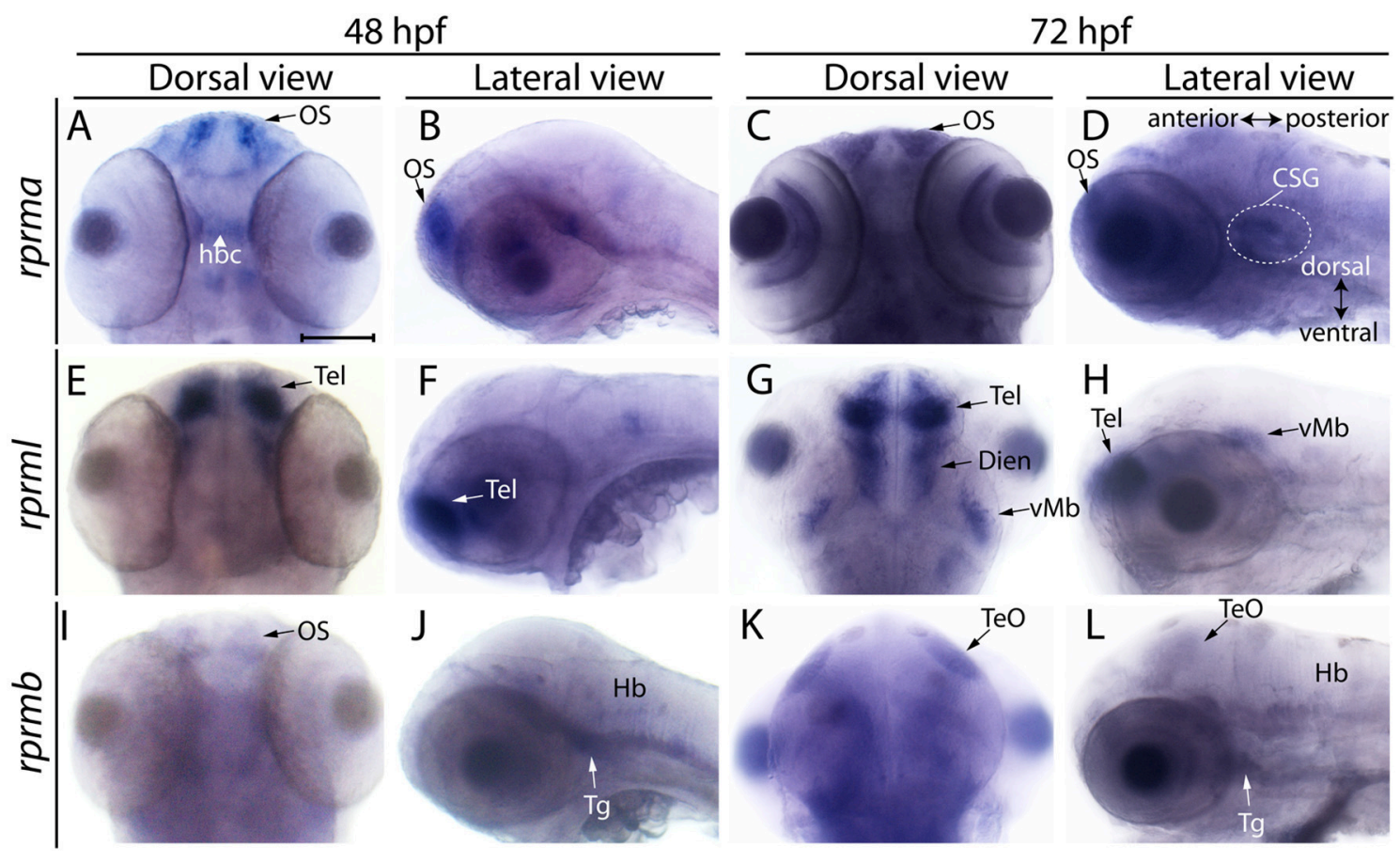

FIGURE 2 | Expression of RPRM mRNA at 48 and 72 hpf in embryonic zebrafish. Expression of RPRM transcripts was examined using WISH in wild-type embryos. At 48 and 72 hpf, (A-D) rprma, (E-H) rprm/ and (I-L) rprmb transcripts are located in specific cell populations. (A,C,E,G,I,K) Dorsal views of the embryos head, with anterior to the top and posterior to the bottom. (B, $\mathbf{D}, \mathbf{F}, \mathbf{H}, \mathbf{J}, \mathbf{L})$ Lateral views of the embryos head with dorsal to the top, ventral to the bottom (double arrow in $\mathbf{D})$, anterior to the left and posterior to the right (double arrow in $\mathbf{D})$. rprma is expressed in the (A-D) olfactory system (OS, black arrows), habenular commissure (hbc, white head arrow) and at 72 hpf in presumptive distal cranial sensory ganglia (CSG, doted circle in $\mathbf{D})$. rprm/ is expressed in the (E-H) Tel (black and white arrows), (G) diencephalon (Dien) and (H) latero-ventral midbrain (vMb). rprmb is expressed in the (I) OS (black arrow), (J) Hb, trigeminal ganglion (Tg, J,L) and (K,L) TeO (black arrows). Scale bar in (A): $100 \mu \mathrm{m}$.

in the anterior surface of the forebrain (FB) corresponding to the olfactory placodes (OP) (Figures $\mathbf{1 A}, \mathbf{B})$. At the same developmental stage, expression of rprml is detected in the telencephalon (Tel) (Figures 1C,D), whereas rprmb is expressed in the tectum opticum ( $\mathrm{TeO}$ ) and the trigeminal ganglia $(\mathrm{Tg})$ with no apparent expression along the FB/Tel (Figures 1E,F).

Between 48 and $72 \mathrm{hpf}$, expression of rprma remains in the olfactory system (OS, Figures 2A-D), habenular commissure (hbc) (Figure 2A) and presumptive cranial sensory ganglions (CSG) (Figure 2D). While rprml mRNA expression was evident in the forebrain, telencephalon and diencephalon and at $72 \mathrm{hpf}$ in the latero-ventral midbrain (vMb) (Figures 2E-H). At $48 \mathrm{hpf}$, weak rprmb expression was detected in the OS, Tg and the hindbrain (Figures 2I,J). At $72 \mathrm{hpf}$, rprmb transcript is now located at the $\mathrm{TeO}$ and $\mathrm{Tg}$ (Figures 2K,L). (Figueroa et al., 2017). The spatiotemporal expression patterns for rprma, rprmb and rprml indicate that these genes are expressed differentially during zebrafish neural development (Figures 1, 2).

\section{RPRM Protein Is Expressed in the OP and OE of Zebrafish Embryos}

Our study, together with previous evidence (Figures 1, 2 and Figueroa et al., 2017), show that the rprma transcript is prominently expressed in the PNS at the OP/OS. To investigate whether RPRM proteins are expressed in different subsets of cells within the peripheral and central nervous system (PNS/CNS), we performed IF coupled with confocal microscopy using a rabbit polyclonal anti-human RPRM antibody, which was generated against an immunogenic 13 amino acidic sequence that is partly conserved between zebrafish and human (Figure 3). By 24 hpf, RPRM is observed in the OP, a restricted domain in the anterior portion of the head (Figures 3A-F). At this developmental stage, RPRM protein also partially overlaps with the axonal marker anti-acetylated tubulin (Ac-tub) in axons projecting from the OP to the developing olfactory bulb (OB) (Figures 3B-C).

\section{Validation of Anti-RPRM Antibody in Zebrafish Embryos}

An alignment of the human and zebrafish RPRM protein sequences revealed a striking conservation pattern on the predicted transmembrane and C-terminus domains of the proteins (Figure 3G), from where the immunogenic sequence comes. This information suggested that anti-human RPRM antibody may also work on zebrafish RPRM proteins. To validate the specificity of the RPRM antibody we performed knockdown of RPRM genes by using antisense oligonucleotide morpholinos (MOs). Reduction in immunoreactivity was 

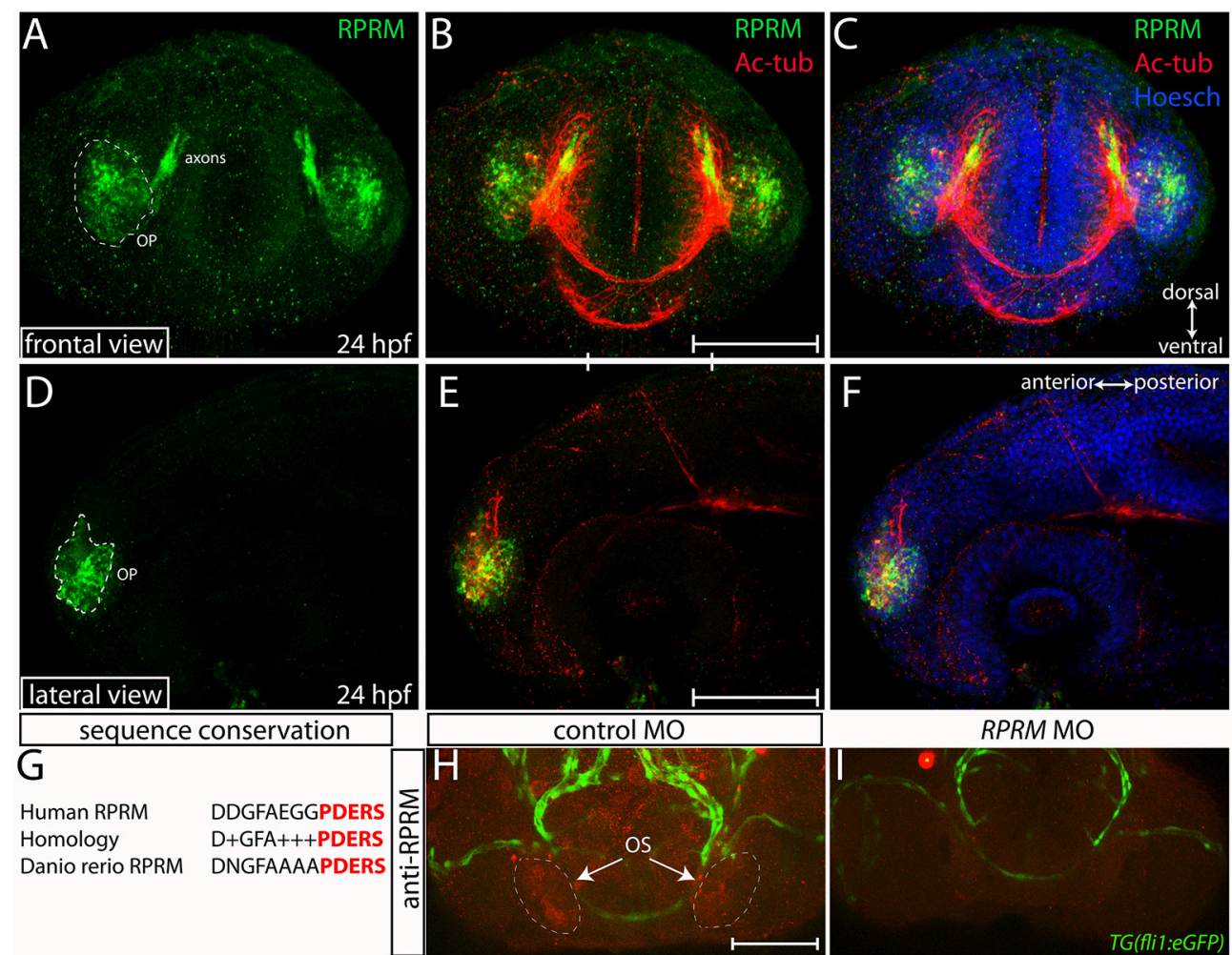

FIGURE 3 | Expression of RPRM protein in the zebrafish olfactory system. RPRM protein localization was examined by immunofluorescence (IF) in wild-type embryos. At $24 \mathrm{~h}$ post-fertilization (hpf). (A-C) Frontal and (D-F) lateral views with dorsal to the top and ventral to the bottom (double arrows in C). (A,D) RPRM is expressed in the OP. The OP give rise to primary sensory neurons, and support basal cells of the OE. (B,E) Overlapped expression of RPRM with acetylated tubulin (Ac-tub) in the axons projecting to the presumptive olfactory bulb (OB) in the central nervous system (CNS). (C,F) Nuclei of the head cells are labeled by Hoechst staining. (G) Sequence conservation between human immunogen and zebrafish RPRM proteins. $(\mathbf{H}, \mathbf{I})$ RPRM protein expression is effectively blocked by antisense oligonucleotide MOs. (H,I) Frontal views of the head region in control-MO and RPRM MO-injected embryos by double immunofluorescence confocal microscopy at $48 \mathrm{hpf}$ (with antibodies against RPRM and GFP). RPRM antibody labeled the olfactory system (OS, in red) in (H) MO-control injected embryos, but not in (I) RPRM MO-injected embryos. Scale bars in (B,E,H): $100 \mu \mathrm{m}$.

confirmed by IHC in MO-injected embryos compared with control MOs (Figures 3H,I). Loss-of-function was performed in the transgenic $T g(f l i 1: G F P)$ line, using the GFP signal as a counter label for immunoreactivity (Figures $3 \mathbf{H}, \mathbf{I}$ ).

Over the following $48 \mathrm{~h}$, RPRM expression maintain restricted to the anterior portion of the PNS (compared Figures 4A-D). At $72 \mathrm{hpf}$, the expression of RPRM is observed in cells adjacent to the apical surface, where the olfactory sensory neurons $\left(\mathrm{OSN}\right.$ ) are located (Figures $\mathbf{4}, \mathbf{F}^{\prime}$, inset magnification), and the supraorbital neuromasts (SO, Figures 4D,F, 5A), distal cranial sensory ganglia (CSG) and the $\mathrm{TeO}$ (Figures $\mathbf{5 A}-\mathbf{C}$ ), showing positive staining in the optic neuropil (Figures 5D-6F), and the axons which project toward the stratum periventriculare (Figures 5E,F). At this developmental stage, RPRM is also located in the habenular commissure (hbc) and in the axons within the hindbrain $(\mathrm{Hb})$ (Figures 6A-C). Our results indicate that in embryonic zebrafish, RPRM is prominently expressed in sensory organs such as the olfactory, optic systems, and ganglia originated from the epibrancheal placodes (Figures 5A-C, $6 \mathrm{~A}-\mathrm{C})$.

\section{Expression of RPRM in the Olfactory System Is Conserved Between Zebrafish and Mouse Embryos}

To test whether expression of RPRM is conserved between zebrafish and mice, we assessed RPRM protein expression in zebrafish at $72 \mathrm{hpf}$ (Figures 6A-C) and mouse RPRM transcript (Figure 6E), and RPRM protein (Figure 6F) at E15.5 developmental stages. These two developmental stages are equivalent when comparing zebrafish and mouse developmental timeline (Kulkeaw and Sugiyama, 2012). In mouse, RPRM mRNA expression was determined by in situ hybridization (ISH) (available data from Allen Brain Atlas, http://mouse.brain-map.org) (Figure 6E) and RPRM gene products were analyzed in the mouse transgenic line $T G(B A C$ 180MB-RPRM-EGFP) derived from a BAC clone (available data from GENSTAT, www.gensat.org; Figures 6F-I). Consistent with our findings in zebrafish, RPRM mRNA is clearly expressed in the OE in mice (E15.5, Figure 6E). Furthermore, enhanced green fluorescent protein (EGFP) expressed under the control of RPRM cis-regulatory modules showed a highly specific and 

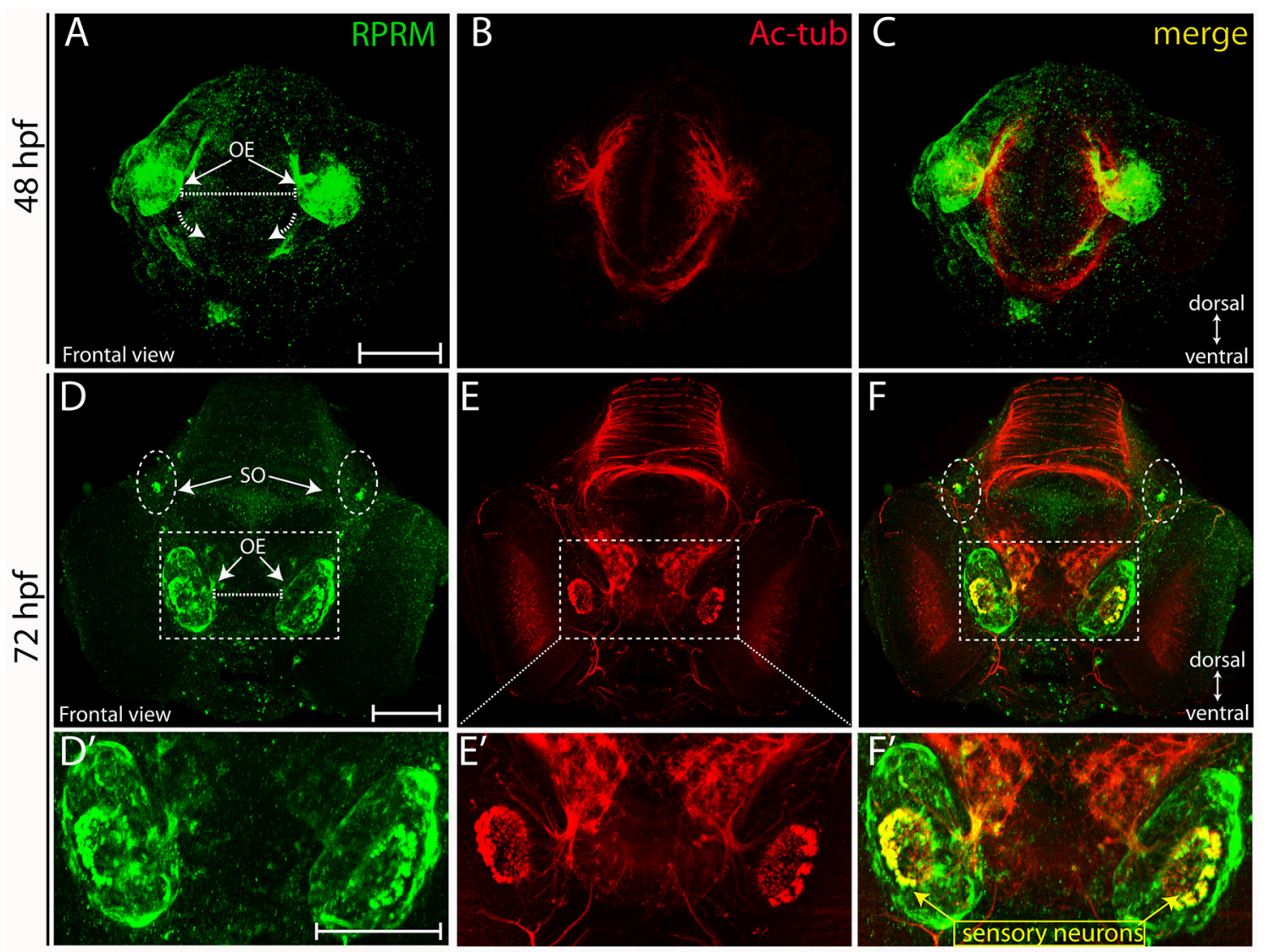

FIGURE 4 | Expression of RPRM in zebrafish olfactory epithelium. RPRM protein expression was analyzed by IF in wild-type embryos. (A-F) Frontal views with dorsal to the top and ventral to the bottom (double arrows in A,D). At 48 hpf (A-C) RPRM is expressed in the OE showing co-localization with Ac-tub. (B-C). At 72 hpf, (D-F) RPRM is expressed at the OE and co-localizes with olfactory sensory neurons (OSNs) which express Ac-tub (F-F') and some of the axons projecting to the OB, also is present in the supraorbital neuromasts (SO) located bilaterally (dotted circles in $\mathbf{D}, \mathbf{F})$. ( $\mathbf{D}^{\prime}-\mathbf{F}^{\prime}$ ) inset magnification at the OE showing RPRM and Ac-tub expression in OSNs (yellow square and arrows). Scale Bars in (A,D, $\mathbf{D}^{\prime}$ ): $100 \mu \mathrm{m}$.

strong EGFP signal in the Mb and OE (Figures 6G,I), while a weak signal was detected in the $\mathrm{OB}$ (Figure 6H). Of note, the EGFP expression is not necessarily a read out for RPRM protein expression. Since EGFP remains expressed even when RPRM is not. Nevertheless, given that this study looks a developmental stages, it is highly likely that the EGFP expression corresponds to the actual protein expression of RPRM. Importantly, the data from the transgenic mouse seems to overlap with the ISH data from the Allen Brian Atlas. Collectively, our findings indicate that expression of RPRM has been conserved throughout the evolution of the vertebrate nervous system.

\section{DISCUSSION}

In order to examine the roles of $R P R M$ genes during the development of the nervous system we performed a detailed neuronal $R P R M$ expression profile in zebrafish and mice. In both species, $R P R M$ transcripts exhibit unique, although partially overlapping localization during neuronal development. Most importantly, the expression patterns for $R P R M$ transcriptional/translational products are highly conserved in both species, as demonstrated by IHC/IF, WISH and transgenic fluorescent reporters. Together, our findings indicate that $R P R M$ genes might possess a pivotal role during neuronal development and that the transcript and protein expression have been conserved during the evolutionary history of vertebrates.

\section{Distinct Expression Patterns Characterize RPRM Gene Products in the Developing Nervous System}

Each RPRM gene, rprma, rprmb and rprml, has very specific expression patterns during neuronal development in zebrafish embryos. The expression of rprma and rprml are largely restricted to defined regions such as $\mathrm{OE}$ and Tel, respectively. Conversely, rprmb transcript is expressed in most posterior neuronal territories such as $\mathrm{TeO}$ (dorsal mesencephalon) and Tg. The observed expression of rprmb mRNA in the $\mathrm{TeO}$ is consistent with RPRM protein expression, detected by IHC/IF, in the zebrafish tectal region (Figures 5A, 6A). Importantly, rprma is the only RPRM gene expressed in the OP and OE, suggesting a 

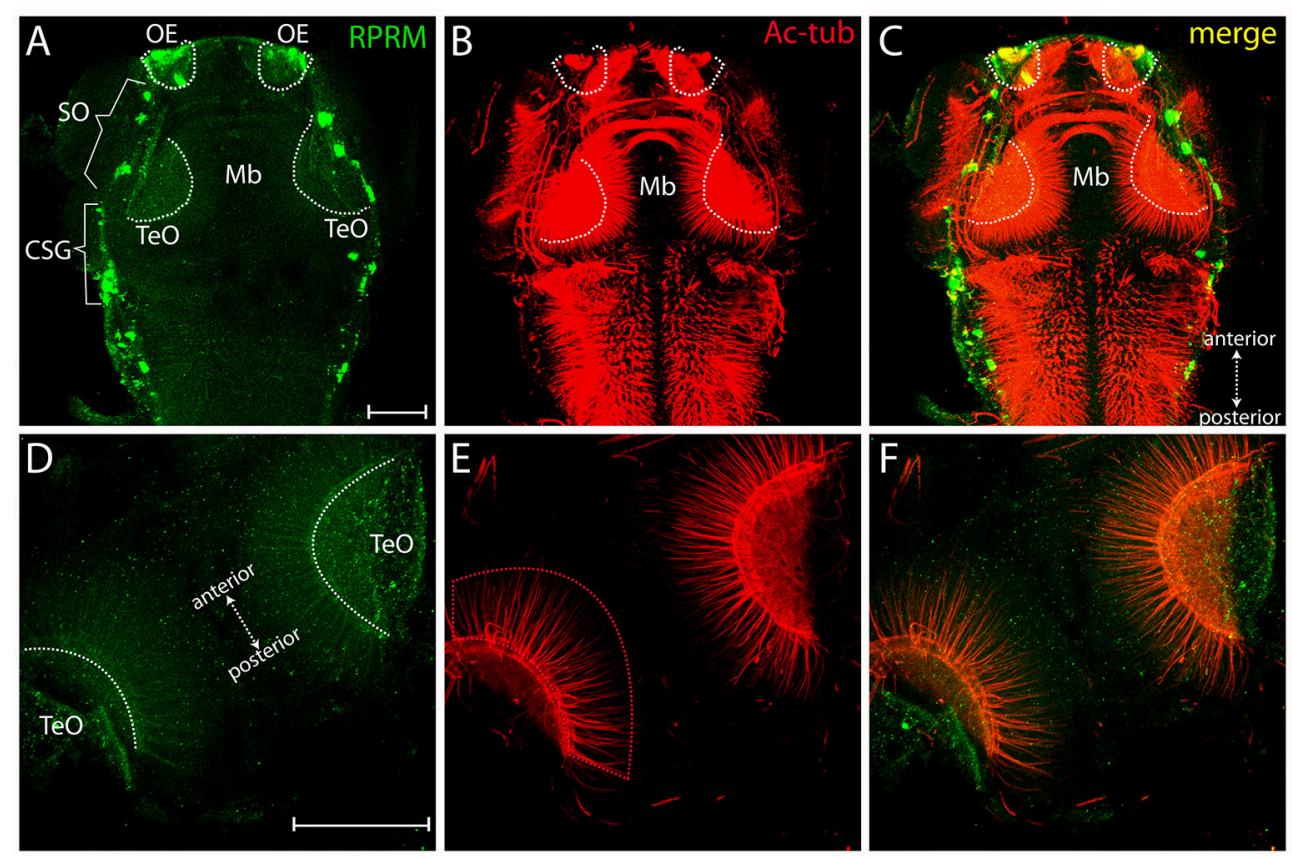

FIGURE 5 | Expression of RRPM in zebrafish tectum opticum. Whole-mount immunofluorescence showing RPRM staining in a representative zebrafish embryo at 3dpf. (A-C) Dorsal view with anterior to the top, posterior to the bottom (double arrows in $\mathbf{C}$ ). Expression of RPRM is shown in green and Ac-tub is shown in red. Positive labeling is observed in the OE, supraorbital neuromasts (SO, brackets), cranial sensory ganglia (CSG, brackets) and TeO (dotted white areas in A-C). (D-F) Magnification of TeO area with anterior to the upper-left and posterior to the bottom-right (double arrows in D), showing optic neuropil area labeled bilaterally (dotted white areas in $\mathbf{D}$ ) as well as projections extending radially toward the midline (red dotted area in $\mathbf{E})$. (F) Merged image for (D,E). (A-F) All the panels represent Z-stack projections. Scale Bars in (A,D): $100 \mu \mathrm{m}$.

putative role for rprma during the development of the olfactory system. In summary, three zebrafish RPRM genes (rprma, rprmb, and $r p r m l$ ) are expressed in different regions of the embryonic PNS and CNS, which indicate a process of subfunctionalization during the evolutionary history of this group of genes and a specific role for each RPRM gene during nervous system development.

\section{RPRM Expression in the Olfactory System}

In vertebrates, the PNS arises fundamentally by tissueinteractions between the neural crest and sensorial placodes (Steventon et al., 2014). In the zebrafish, the OPs appear as thickenings at the anterior ectoderm by 17-18 hpf and these thickenings later invaginate to form the $\mathrm{OE}$ covering the opening of the nose, by $32 \mathrm{hpf}$ (Hansen and Zeiske, 1993). Here we show that Rprm protein is located in the OP and that expression in this territory is maintained throughout specification of the OE (Figures 3, 4). At $24 \mathrm{hpf}$, a unique subset of pioneer axons projects caudally from the OP to form the olfactory nerve and establishes glomerular-like structures in the presumptive $\mathrm{OB}$ (Whitlock and Westerfield, 1998). The axons we have detected using an antibody against RPRM seem to be pioneer axons (Figure 3), as no other projections exit from the OP at this developmental stage. Interestingly, the $\mathrm{OE}$ is one of the few tissues that present neurogenesis during adulthood due to the persistence of a pool of embryonic neuronal stem cells (NSCs)
(Madelaine et al., 2011). Previously, RPRM has been described as regulator of cell cycle (Ohki et al., 2000), thus RPRM could potentially play a role during self-renewal of NSC in the adult olfactory system.

\section{Comparison of RPRM Gene Expression Patterns Between Zebrafish and Mice}

Our previous studies in human revealed that RPRM protein was expressed in the brain (Figueroa et al., 2017). Moreover, mice embryos showed strong $R P R M$ transcriptional expression in the $\mathrm{OE}$, using ISH and/or the mouse reporter transgenic line $T G(B A C-180 M B-R P R M-E G F P)$. This expression pattern is consistent with the mRNA and protein localization observed in the olfactory system of zebrafish embryos (Figure 6). In mice, $R P R M$ is broadly expressed in the habenular nuclei, $\mathrm{Mb}$ and $\mathrm{Hb}$; in accordance with $R P R M$ expression pattern in embryonic zebrafish. Altogether, our findings indicate that the conserved expression of the RPRM gene family through vertebrates might implicate an essential role for $R P R M$ during neural development. Future mechanistic studies will be required to clarify $R P R M$ function in neuronal tissues.

\section{Evolutionary Role of the RPRM Gene Family}

Reprimo genes seem to be an evolutionary innovation of vertebrates as no traces of them have been annotated in 

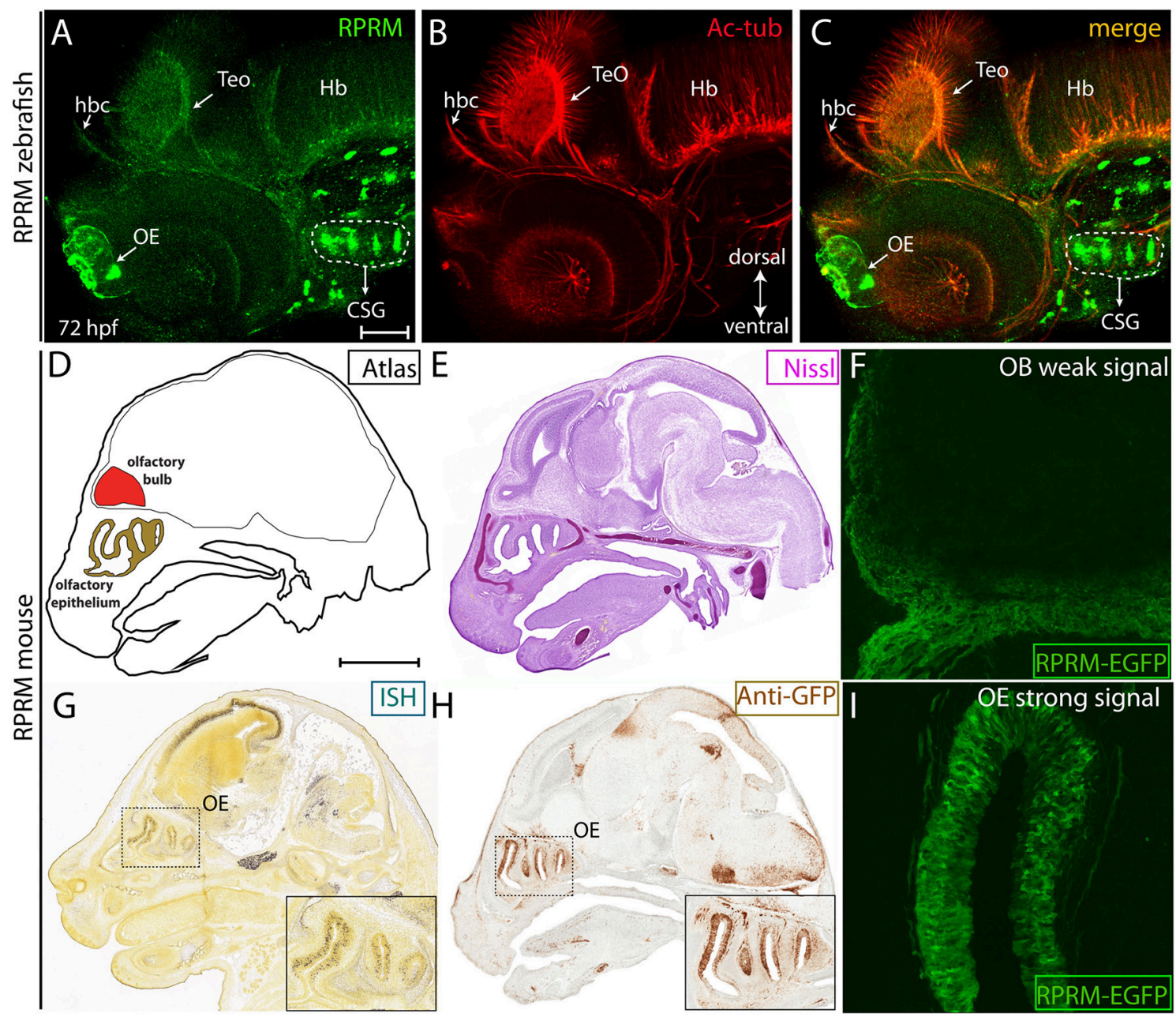

FIGURE 6 | RPRM expression is conserved between zebrafish and mice. (A-I) Zebrafish RPRM protein expression, visualized by IF at 72 hpf, presents similar expression patterns when compared with murine RPRM in comparable developmental stages. (A-C) Lateral view of a representative 72 hpf embryo by IF, showing RPRM (green) and Ac-tub (red) expression in the habenular commissure (hbc), OE, TeO, CSG, and hindbrain (Hb). Dorsal is show to the top and ventral to the bottom (double arrow in B) (D) Sagittal atlas image of E15.5 mouse embryo showing reference areas of the OS (OE, OB). (E) Nissl staining of sagittal section from E15.5 mouse embryo. (G,H) RPRM expression as detected by in situ hybridization (ISH) and (F) immunohistochemistry (IHC) for anti-GFP in the transgenic mice line TG(BAC-180MB-RPRM-EGFP). (F-I) RPRM expression pattern in TG(BAC-180MB-RPRM-EGFP) sections, showing low levels of RPRM gene products in the OB/OS (F) and high levels of expression in the OE (I). Scale Bars in (A): $100 \mu \mathrm{m}$, in (D): $1 \mathrm{~mm}$.

other taxa (Wichmann et al., 2016). This group of genes diversified as a consequence of the two rounds of whole genome duplications occurred early in the evolutionary history of vertebrates (Wichmann et al., 2016). In teleost fish (e.g., zebrafish, Danio rerio) the repertoire of RPRM genes further expanded as a consequence of the teleost-specific genome duplication (Meyer and Van de Peer, 2005; Kasahara, 2007). Thus, current species of teleost fish possess a repertoire of four reprimo genes: rprma, rprmb, rprml, and rprm3 (Wichmann et al., 2016). Transcriptome studies in representative species of vertebrates have shown that $R P R M$ genes are mostly expressed in the CNS, and that this pattern has been maintained for more than 500 million years during the evolutionary history of the group (Wichmann et al., 2016). Although RPRM genes have a function during the cell cycle and the neurons in general does not experience cell division, their expression in the adult nervous tissues could be seen as a protective measure within this organ (Zohrabian et al., 2007). More recently, we determined the $R P R M$ expression profiles during embryonic development using zebrafish as a model system (Figueroa et al., 2017). In agreement with our in silico analysis, $R P R M$ genes are expressed in the developing brain but also in digestive tube and blood vessels (Figueroa et al., 2017). In the present study we found that within the PNS, RPRM is mainly expressed in the $\mathrm{OE}$ and that this pattern is shared with mammals. The similarity in the RPRM expression pattern between both groups is consistent with the fact that at the anatomical level the general organization of the olfactory system is conserved among vertebrates (Kermen et al., 2013; Saraiva et al., 2015). The olfactory epithelium (OE) of bony fish, where 
reprimo genes are mostly expressed, is also conserved among vertebrates.

At the amino acid level, there is a striking conservation throughout the C-terminus of the RPRM family, suggesting a strong selective pressure of this domain that could be related to the functional role of this protein family. The conservation of the amino acidic sequences between zebrafish and human $R P R M$ proteins, as well as the conservation of the expression pattern of these genes, suggest that $R P R M$ proteins might play similar roles in both groups.

Due to the highly specific and conserved $R P R M$ expression in the brain and specific sensory organs in different vertebrates, $R P R M$ genes might play a pivotal role during sensory neurons development, such as olfactory receptors as well as other detected sensory system structures like SO and CSG, all originating from neural crest and ectodermal placodes. With our present study, we provide novel knowledge for future investigation directed to determine the physiological functions of $R P R M$ in vertebrate $\mathrm{PNS} / \mathrm{CNS}$, from the cellular level to animal behavior.

\section{AUTHOR CONTRIBUTIONS}

KS and AQ: conceptualization, data curation, formal analysis, validation, methodology, writing (editing); CL: data curation;
IW, AC, and GO: writing (editing); JO: conceptualization, formal analysis, writing (editing); MC: conceptualization, formal analysis, writing (editing), funding acquisition. JA: conceptualization, data curation, formal analysis, validation, methodology, funding acquisition, project administration, supersision, writing (editing), writing (editing, original draft).

\section{FUNDING}

BMBF-CONICYT 20140027 to JA. CONICYT-ANILLO ACT1402 to MC and JA. CRP-ICGEB CHL15-01 to JA. FONDECYT 1160627 to JO. FONDECYT 1140970 to GO. IMII P09/016-F to GO. CONICYT-FONDAP 15130011 to AC and GO. FONDECYT 1151411 to AC.

\section{ACKNOWLEDGMENTS}

We would like to thank Allen Brain Atlas Data Portal (www.mouse.brain-map.org) and GENSAT (Gene Expression Nervous System Atlas, www.gensat.org) for kindly providing us with the images from RPRM WISH and transgenic reporter RPRM-EGFP in mice. We also wish to thank to Ricardo Figueroa for technical assistance and all members of Amigo's lab for the critical review of this manuscript.

\section{REFERENCES}

Amigo, J. D., Ackermann, G. E., Cope, J. J., Yu, M., Cooney, J. D., Ma, D., et al. (2009). The role and regulation of friend of GATA-1 (FOG-1) during blood development in the zebrafish. Blood 114, 4654-4663. doi: 10.1182/blood-2008-12-189910

Figueroa, R. J., Carrasco-Avino, G., Wichmann, I. A., Lange, M., Owen, G. I., Siekmann, A. F., et al. (2017). Reprimo tissue-specific expression pattern is conserved between zebrafish and human. PLoS ONE 12:e0178274. doi: 10.1371 /journal.pone. 0178274

Hansen, A., and Zeiske, E. (1993). Development of the olfactory organ in the zebrafish, Brachydanio rerio. J. Comp. Neurol. 333, 289-300. doi: 10.1002/cne. 903330213

Hildebrand, D. G. C., Cicconet, M., Torres, R. M., Choi, W., Quan, T. M., Moon, J., et al. (2017). Whole-brain serial-section electron microscopy in larval zebrafish. Nature 545, 345-349. doi: 10.1038/nature22356

Kasahara, M. (2007). The 2R hypothesis: an update. Curr. Opin. Immunol. 19, 547-552. doi: 10.1016/j.coi.2007.07.009

Katoh, K., and Standley, D. M. (2013). MAFFT multiple sequence alignment software version 7: improvements in performance and usability. Mol. Biol. Evol. 30, 772-780. doi: $10.1093 / \mathrm{molbev} / \mathrm{mst} 010$

Kermen, F., Franco, L. M., Wyatt, C., and Yaksi, E. (2013). Neural circuits mediating olfactory-driven behavior in fish. Front. Neural Circuits 7:62. doi: $10.3389 /$ fncir.2013.00062

Kimmel, C. B., Ballard, W. W., Kimmel, S. R., Ullmann, B., and Schilling, T. F. (1995). Stages of embryonic development of the zebrafish. Devel. Dyn. 203, 253-310. doi: 10.1002/aja.1002030302

Kulkeaw, K., and Sugiyama, D. (2012). Zebrafish erythropoiesis and the utility of fish as models of anemia. Stem Cell Res. Ther. 3:55. doi: 10.1186/scrt146

MacRae, C. A., and Peterson, R. T. (2015). Zebrafish as tools for drug discovery. Nat. Rev. Drug Discov. 14, 721-731. doi: 10.1038/nr $\mathrm{d} 4627$

Madelaine, R., Garric, L., and Blader, P. (2011). Partially redundant proneural function reveals the importance of timing during zebrafish

olfactory neurogenesis. Development $138,4753-4762$. doi: 10.1242/dev.0 66563

Meyer, A., and Van de Peer, Y. (2005). From 2R to 3R: evidence for a fishspecific genome duplication (FSGD). Bioessays 27, 937-945. doi: 10.1002/bies. 20293

Ohki, R., Nemoto, J., Murasawa, H., Oda, E., Inazawa, J., Tanaka, N., et al. (2000). Reprimo, a new candidate mediator of the p53-mediated cell cycle arrest at the G2 phase. J. Biol. Chem. 275, 22627-22630. doi: 10.1074/jbc.C0002 35200

Saavedra, K., Valbuena, J., Olivares, W., Marchant, M. J., Rodríguez, A., Torres-Estay, V., et al. (2015). Loss of expression of reprimo, a p53induced cell cycle arrest gene, correlates with invasive stage of tumor progression and p73 expression in gastric cancer. PLOS ONE 10:e0125834. doi: 10.1371/journal.pone.0125834

Saraiva, L. R., Ahuja, G., Ivandic, I., Syed, A. S., Marioni, J. C., Korsching, S. I., et al. (2015). Molecular and neuronal homology between the olfactory systems of zebrafish and mouse. Sci. Rep. 5:11487. doi: 10.1038/srep 11487

Sato, N., Fukushima, N., Matsubayashi, H., Iacobuzio-Donahue, C. A., Yeo, C. J., and Goggins, M. (2006). Aberrant methylation of Reprimo correlates with genetic instability and predicts poor prognosis in pancreatic ductal adenocarcinoma. Cancer 107, 251-257. doi: 10.1002/cncr. 21977

Steventon, B., Mayor, R., and Streit, A. (2014). Neural crest and placode interaction during the development of the cranial sensory system. Dev. Biol. 389, 28-38. doi: 10.1016/j.ydbio.2014.01.021

Taylor, W. R., and Stark, G. R. (2001). Regulation of the G2/M transition by p53. Oncogene 20, 1803-1815. doi: 10.1038/sj.onc. 1204252

Westerfield, M. (1995). The Zebrafish Book, 3rd Edn. Eugene, OR: University of Oregon Press.

Whitlock, K. E., and Westerfield, M. (1998). A transient population of neurons pioneers the olfactory pathway in the zebrafish. J. Neurosci. 18, 8919-8927.

Wichmann, I. A., Zavala, K., Hoffmann, F. G., Vandewege, M. W., Corválan, A. H., Amigo, J. D., et al. (2016). Evolutionary history of the reprimo 
tumor suppressor gene family in vertebrates with a description of a new reprimo gene lineage. Gene 591, 245-254. doi: 10.1016/j.gene.2016.0 7.036

Xu, M., Knox, A. J., Michaelis, K. A., Kiseljak-Vassiliades, K., KleinschmidtDeMasters, B. K., Lillehei, K. O., et al. (2012). Reprimo (RPRM) is a novel tumor suppressor in pituitary tumors and regulates survival, proliferation, and tumorigenicity. Endocrinology 153, 2963-2973. doi: 10.1210/en.201 $1-2021$

Zohrabian, V. M., Nandu, H., Gulati, N., Khitrov, G., Zhao, C., Mohan, A., et al. (2007). Gene expression profiling of metastatic brain cancer. Oncol. Rep. 18, 321-328. doi: 10.3892/or.18.2.321
Conflict of Interest Statement: The authors declare that the research was conducted in the absence of any commercial or financial relationships that could be construed as a potential conflict of interest.

Copyright (๔ 2018 Stanic, Quiroz, Lemus, Wichmann, Corvalán, Owen, Opazo, Concha and Amigo. This is an open-access article distributed under the terms of the Creative Commons Attribution License (CC BY). The use, distribution or reproduction in other forums is permitted, provided the original author(s) and the copyright owner are credited and that the original publication in this journal is cited, in accordance with accepted academic practice. No use, distribution or reproduction is permitted which does not comply with these terms. 